\title{
As borboletas (Lepidoptera: Papilionoidea e Hesperioidea) da Área de Proteção Ambiental do Gama e Cabeça de Veado (Distrito Federal, Brasil)
}

\author{
Carlos Eduardo Guimarães Pinheiro' ${ }^{1}$ Eduardo de Oliveira Emery ${ }^{2}$
}

Biota Neotropica v6 (n3)-http://www.biotaneotropica.org.br/v6n3/pt/abstract?inventory+bn01506032006

\author{
Recebido em 10/05/06. \\ Versão reformulada recebida em 07/08/06. \\ Publicado em 08/09/06
}

\begin{abstract}
${ }^{1}$ Departamento de Zoologia, IB, Universidade de Brasília - UnB, 70910-900 Brasília, DF, Brasil. E-mail: cegp@unb.br ${ }^{2}$ Curso de pós-graduação em Biologia Animal, Instituto de Biologia, Universidade de Brasília - UnB, CEP 70910-900, Brasília, DF, Brasil. E-mail: eoemery@unb.br
\end{abstract}

\begin{abstract}
Pinheiro, C.E.G. and Emery, E. O. The butterflies (Lepidoptera: Papilionoidea and Hesperioidea) of the Environmental Protection Zone/APA do Gama e Cabeça de Veado, Distrito Federal, Brazil. Biota Neotrop. Sep/Dec 2006 vol. 6, no. 3 http://www.biotaneotropica.org.br/v6n3/pt/abstract?inventory+bn01506032006. ISSN 1676-0603
\end{abstract}

A list containing 507 species of butterflies (Papilionoidea e Hesperioidea) found in the APA do Gama e Cabeça de Veado - DF in the last four decades is presented in this study. The list includes data obtained in the literature, our personal collections, and entomological collections recently visited, but not species with presumed distribution in the region. The purpose of this study is (1) to demonstrate the importance of this APA for the conservation of the cerrado butterfly fauna and, (2) allow for monitoring such biodiversity in future studies.

Key words: butterflies, Cerrado, conservation, biodiversity, inventory.

\section{Resumo}

Pinheiro, C.E.G. and Emery, E. O. As borboletas (Lepidoptera: Papilionoidea e Hesperioidea) da Área de Proteção Ambiental do Gama e Cabeça de Veado, Distrito Federal, Brasil. Biota Neotrop. Sep/Dec 2006 vol. 6, no. 3 http:// www.biotaneotropica.org.br/v6n3/pt/abstract?inventory+bn01506032006. ISSN 1676-0603

Uma listagem atualizada com 507 espécies de borboletas (Papilionoidea e Hesperioidea) encontradas na APA do Gama e Cabeça de Veado - DF nas últimas quatro décadas é apresentada neste estudo. Foram reunidos dados da bibliografia, de nossas coletas pessoais e coleções entomológicas, mas espécies com ocorrência apenas presumida não foram incluídas. Nossos objetivos com esta publicação são: (1) demonstrar a importância desta APA para a conservação da fauna de borboletas do cerrado e (2) permitir o monitoramento futuro da biodiversidade deste grupo de insetos nestes locais.

Palavras-chave: borboletas, Cerrado, conservação, biodiversidade, inventário.

http://www.biotaneotropica.org.br 


\section{Introdução}

A Área de Proteção Ambiental (APA) das bacias do Gama e Cabeça de Veado foi criada através do Decreto Distrital no 9.417 de 21 de abril de 1986, com o objetivo de proteger as cabeceiras do ribeirão do Gama e do córrego Cabeça de Veado, de forma a garantir a integridade dessas drenagens, responsáveis por um terço das águas do Lago Paranoá e pelo abastecimento de água potável para parte da população do Distrito Federal.

Com uma área aproximada de 25.000 ha, a APA se localiza na área nuclear do bioma do cerrado e contêm algumas das principais fisionomias deste tipo de vegetação, especialmente o cerrado sensu stricto, campos cerrados, cerradões e matas de galeria (Eiten 1972). Nesta região estão incluídas importantes instituições de pesquisa científica e conservação, como a Estação Ecológica do Jardim Botânico (E. E. J. B.; 5.000 ha), a Reserva Ecológica do IBGE (1.360 ha), a Fazenda Experimental Água Limpa da Universidade de Brasília (F. A. L.; 4.040 ha), o Jardim Zoológico de Brasília e várias pequenas Áreas de Relevante Interesse Ecológico (ARIE), como o Santuário de Vida Silvestre do Riacho Fundo, Capetinga-Taquara e Cerradão, além do Aeroporto Internacional de Brasília e várias áreas urbanas e rurais. Uma grande variedade de estudos científicos tem sido desenvolvida nesta APA nas últimas décadas, tornando-a uma das áreas de cerrado mais conhecidas em termos ecológicos, zoológicos e botânicos (Henriques et al. 1999).

O primeiro estudo aprofundado envolvendo a fauna de borboletas desta região foi realizado por Brown \& Mielke (1967a,b) em áreas vizinhas à APA, como o Jardim Zoológico de Brasília, o Parque do Gama (143 ha) e o Brasília Country Clube. Outros trabalhos envolvendo levantamentos da fauna de borboletas foram realizados por Ferreira (1982), Pinheiro \& Ortiz (1992), Diniz \& Morais (1995, 1997)e Pinheiro (2005). Entretanto, uma listagem atualizada e mais abrangente das espécies da APA, tão necessária para o monitoramento da biodiversidade local e outros estudos sobre a biologia destes insetos ainda não foi produzida. A produção desta lista, incluindo apenas as espécies efetivamente observadas ou coletadas no local, constitui o propósito deste estudo, na esperança de que estudos futuros envolvendo o monitoramento destas espécies na APA possam ser desenvolvidos.

\section{Material e Métodos}

A lista de espécies apresentada a seguir foi baseada nos levantamentos de fauna realizados por Brown \& Mielke (1967a,b) no Jardim Zoológico de Brasília, Ferreira (1982) na E. E. J. B. e Reserva do IBGE, Pinheiro \& Ortiz (1992) na E. E. J. B., Diniz \& Morais $(1995,1997)$ na F.A. L. e Reserva do IBGE, além de várias coletas realizadas pelos autores (dados não pub.) em diferentes localidades na APA e dados obtidos nas Coleções Entomológicas da Universidade de Brasília e do IBGE. Espécies com distribuições geográficas apenas presumidas não foram incluídas. A nomenclatura adotada segue Lamas (2004).

\section{Resultados e Discussão}

No total 507 espécies de Papilionoidea e Hesperioidea foram registradas na área da APA (ver Tabela 1). Este número de espécies corresponde a mais da metade de todas as espécies de borboletas do Distrito Federal (Emery et al. 2006), demonstrando assim a grande diversidade de espécies aí encontrada. Entretanto, podemos esperar que o número de espécies que aí ocorrem seja de fato maior, por várias razões. Em primeiro lugar, várias espécies de Satyrinae (Nymphalidae), Lycaenidae, Riodinidae e Hesperiidae registradas por Ferreira (1982) foram omitidas desta lista, em vista da ausência de espécimes depositadas pela autora em coleções científicas e as grandes modificações ocorridas recentemente na classificação e taxonomia destes grupos. Em segundo lugar, a ocorrência de novos registros, principalmente nas famílias citadas acima, ainda tem sido obtida quando novas amostragens são realizadas nestas áreas. Finalmente, o fato de incluirmos apenas as espécies efetivamente observadas ou coletadas nestas áreas também indica que este número é conservador, pois várias espécies de borboletas com distribuição geográfica mais ampla também poderiam aí ocorrer. A lista inclui ainda uma espécie e uma subespécie consideradas como ameaçadas de extinção no estado de Minas Gerais: Magnastigma julia (Theclinae, Lycaenidae) e Agrias claudina godmani (Charaxinae, Nymphalidae), a borboleta mais procurada por colecionadores (Brown et al. 1988). A ocorrência destas borboletas ainda recentemente observadas na E. E. J. B., na Reserva do IBGE e F.A.L., demonstra o bom estado de conservação da vegetação local e a importância destas áreas para a preservação da fauna de borboletas do cerrado. Por outro lado, o avanço da urbanização e de várias outras atividades econômicas desenvolvidas nesta região vem transformando a APA em uma verdadeira "ilha de vegetação”, geograficamente isolada de outras unidades de conservação. Os efeitos advindos do isolamento sobre as populações locais, como a interrupção do fluxo gênico, pode ser fatal para a grande maioria das espécies. Do mesmo modo, a área da APA está sujeita a constantes incêndios e a presença de depósitos de lixo, animais domésticos, caçadores clandestinos e plantas invasoras. Esperamos que medidas político-administrativas eficazes sejam rapidamente tomadas para que toda esta riqueza em biodiversidade seja de fato preservada.

\section{Agradecimentos}

Somos gratos a Keith S. Brown Jr., André V. L. Freitas, Olaf H. H. Mielke, Paulo C. Motta, Ivone R. Diniz, Helena C. Morais e Carla M. Penz pela cooperação, troca de informações ou identificação de espécies. 


\section{Referências Bibliográficas}

Brown Jr., K.S. \& Mielke, O.H.H. 1967a. Lepidoptera of the Central Brazil Plateau. I. Preliminary list of Rhopalocera: Introduction, Nymphalidae, Libytheidae. J. Lepid. Soc. 21: 77-106.

Brown Jr., K.S. \& Mielke, O.H.H. 1967b. Lepidoptera of the Central Brazil Plateau. II. Preliminary list of Rhopalocera (continued): Lycaenidae, Pieridae, Papilionidae, Hesperiidae. J. Lepid. Soc. 21: 145-168.

Brown Jr., K.S., Mielke, O.H.H. \& Casagrande, M.M. 1988. Espécies de Lepidoptera ameaçadas do Estado de Minas Gerais. In Livro vermelho das espécies ameaçadas de extinção da fauna de Minas Gerais (A.B.M. Machado; G.A.B. Fonseca; Machado, R.B.; Aguiar, L.M.S. \& Lins, L.V. eds.). Fundação Biodiversitas, Belo Horizonte, p. 512-559.

Diniz, I.R. \& Morais, H.C. 1995. Larvas de Lepidoptera e suas plantas hospedeiras em um cerrado de Brasília, Distrito Federal, Brasil. Rev. Bras. Entom. 39: 755-770.

Diniz, I.R. \& Morais, H.C. 1997. Lepidopteran caterpillar fauna of cerrado host plants. Biodiv. Conserv. 6: 817-836.

Eiten, G. 1972. The cerrado vegetation of central Brazil. Bot. Rev. 38: 205-341.

Emery, E.O., Brown Jr., K.S. \& Pinheiro, C.E.G. 2006. As borboletas (Lepidoptera, Papilionoidea) do Distrito Federal, Brasil. Rev. Bras. Entom. 50: 85-92.

Ferreira, L.M. 1982. Comparações entre riqueza, diversidade e equitabilidade de borboletas em três áreas com diferentes graus de perturbação, próximas a Brasília. Tese de Mestrado, Brasília. Universidade de Brasília.

Henriques, R.P.B., Morais, H.C. \& Palma, A.R.T. 1999. Bibliografia dos cerrados da APA do Gama e Cabeça de Veado.http://www.unb.br/ib/ecl/posecl/ bibliografiacerrado.htm 10/03/2006.

Lamas, G. (ed.) 2004. Checklist: Part 4A. Hesperioidea Papilionoidea. In Atlas of Neotropical Lepidoptera. Volume 5A(J.B. Heppner, ed.). Association for Tropical Lepidoptera / Scientific Publishers, Gainesville.

Pinheiro, C.E.G. 2005. Estudos comparativos sobre a fauna de borboletas do Distrito Federal: Implicações para a conservação. In Biodiversidade, Ecologia e Conservação do Cerrado (Scariot, A., Silva, J.C.S. \& Felfili J.M. eds.). MMA, Brasília.

Pinheiro, C.E.G. \& Ortiz, J.V.C. 1992. Communities of fruitfeeding butterflies along a vegetation gradient in central Brazil. J. Biogeog. 19: 505-511.
Título: As borboletas (Lepidoptera: Papilionoidea e Hesperioidea) da Área de Proteção Ambiental do Gama e Cabeça de Veado, Distrito Federal, Brasil.

Autores: Pinheiro, C.E.G. \& Emery, E. O.

Biota Neotropica, Vol. 6 ( número 3 ): 2006

http://www.biotaneotropica.org.br/v6n3/pt/ abstract?inventory+bn01506032006

Recebido em 10/05/06 - Versão reformulada recebida em 07/08/06 - Publicado em 08/09/06

ISSN 1676-0603 
TABELA 1. Lista de borboletas (Hesperioidea e Papilionoidea) da APA do Gama e Cabeça de Veado, DF, Brasil.

Table 1 - List of butterflies (Hesperioidea and Papilionoidea) from the Environmental Protection Zone/APA do Gama e Cabeça de Veado, DF, Brazil.

FAMÍLIA

HESPERIIDAE Pyrrhopyginae/Pyrrhopygini

Pyrginae/Eudamini
ESPÉCIES

Pyrrhopyge charybdis charybdis Westwood, 1852

Pyrrhopyge pelota Plötz, 1879

Pyrrhopyge sergius ssp.

Elbella azeta giffordi Mielke, 1995

Elbella intersecta losca Evans, 1951

Elbella luteizona (Mabille, 1877)

Jemadia menechmus (Mabille,1878)

Mimoniades versicolor versicolor (Latreille, [1824])

Mysoria barcastus barta Evans, 1951

Microceris variicolor (Ménétriés, 1855)

Phocides polybius phanias (Burmeister, 1880)

Udranomia kikkawai (Weeks, 1906)

Udranomia spitzi (Hayward, 1942)

Proteides mercurius mercurius (Fabricius, 1787)

Chioides catillus catillus (Cramer, 1779)

Aguna albistria albistria (Plötz, 1880)

Aguna asander asander (Hewitson, 1867)

Aguna aurunce hypozonius (Plötz, 1880)

Typhedanus undulatus (Hewitson, 1867)

Polythrix octomaculata octomaculata (Sepp, [1844])

Urbanus cindra Evans, 1952

Urbanus dorantes dorantes (Stoll, 1790)

Urbanus doryssus doryssus (Swainson, 1831)

Urbanus esta Evans, 1952

Urbanus evenus (Ménétriés,1855)

Urbanus procne (Plötz, 1880)

Urbanus proteus proteus (Linnaeus, 1758)

Urbanus simplicius (Stoll, 1790)

Urbanus teleus (Hübner, 1821)

Epargyreus exadeus exadeus (Cramer, 1779)

Astraptes fulgerator fulgerator (Walch, 1775)

Astraptes talus (Cramer, 1777)

Autochton integrifascia (Mabille, 1891)

Autochton itylus Hübner, 1823

Autochton neis (Geyer, 1832)

Autochton zarex (Hübner, 1818)

Bungalotis erythus (Cramer, 1775)

Sarmientoia almeidai Mielke, 1967

Celaenorrhinus similis Hayward, 1933

http://www.biotaneotropica.org.br 
Pyrginae/Pyrgini
Spathilepia clonius (Cramer, 1775)

Cogia calchas (Herrich-Schäffer, 1869)

Cogia cerradicola (Mielke, 1967)

Cogia grandis Riley, 1921

Cogia hassan evansi Bell, 1937

Telemiades amphion misitheus (Mabille, 1878)

Sophista latifasciata latifasciata (Spitz, 1930)

Nisoniades castolus (Hewitson, 1878)

Nisoniades macarius (Herrich-Schäffer, 1870)

Pachyneuria inops (Mabille, 1877)

Viola violella (Mabille, 1898)

Staphylus melangon epicaste Mabille, 1903

Trina geometrina geometrina (C. Felder \& R. Felder, 1867)

Gorgythion begga begga (Prittwitz, 1868)

Gorgythion beggina escalophoides Evans, 1953

Gorgythion canda Evans, 1953

Ouleus fridericus candangus Mielke, 1968

Zera hyacinthinus ssp.

Quadrus cerialis (Stoll, 1782)

Quadrus jacobus (Plötz, 1884)

Quadrus u-lucida ssp.

Gindanes brontinus bronta Evans, 1953

Pythonides jovianus fabricii Kirby, 1871

Sostrata bifasciata bifasciata Ménétriés, 1829

Mylon pelopidas (Fabricius, 1793)

Clito bibulus (Riley, 1929)

Clito sompa Evans, 1953

Xenophanes tryxus (Stoll, 1780)

Antigonus erosus (Hübner, [1812])

Antigonus liborius liborius Plötz, 1884

Zopyrion evenor evenor Godman 1901

Zopyrion reticulata Hayward, 1942

Anisochoria pedaliodina extincta Hayward, 1933

Anisochoria sublimbata Mabille, 1883

Achlyodes mithridates thraso (Hübner, [1807])

Grais stigmaticus stigmaticus (Mabille, 1883)

Timochares trifasciata trifasciata (Hewitson, 1868)

Anastrus sempiternus simplicior (Möschler, 1877)

Cycloglypha pollax Evans, 1953

Cycloglypha sp.

Cycloglypha thrasibulus thrasibulus (Fabricius, 1793)

Helias phalaenoides palpalis (Latreille, [1824])

Theagenes dichrous (Mabille, 1878)

Chiomara asychis autander (Mabille, 1891)

Chiomara basigutta (Plötz, 1884)

Gesta austerus (Schaus, 1902)

Gesta gesta (Herrich-Schäffer, 1863)

Gesta heteropterus (Plötz, 1884)

Erynnis (Erynnides) funeralis (Scudder \& Burgess, 1870)

Pyrgus orcus (Stoll, 1780)

Heliopetes alana (Reakirt, 1868) 
Heliopetes arsalte (Linnaeus, 1758)

Heliopetes macaira orbigera (Mabille, 1888)

Heliopetes omrina (Butler, 1870)

Hesperiinae

Zariaspes mys (Hübner, [1808])

Anthoptus epictetus (Fabricius, 1793)

Anthoptus insignis (Plötz, 1882)

Corticea lysias potex Evans, 1955

Apaustus gracilis ssp. $\mathrm{n}$.

Callimormus corades (C. Felder, 1862)

Callimormus juventus Scudder, 1872

Callimormus radiola pusillus Hayward, 1934

Callimormus saturnus (Herrich-Schäffer, 1869)

Methionopsis ina (Plötz, 1882)?

Sodalia coler (Schaus, 1902)

Artines acroleuca Plötz, 1884

Artines bipunctata Mielke, 1968

Mnaseas bicolor inca Bell, 1930

Lucida lucia lucia (Capronnier, 1874)

Vidius felus Mielke, 1968

Vidius spitzi Mielke, 1967

Vidius vidius (Mabille, 1891)

Cymaenes alumna (Butler, 1877)

Cymaenes chela Evans, 1955

Cymaenes gisca Evans, 1955

Cymaenes lepta (Hayward, 1939)

Cymaenes tripunctus theogenis (Capronnier, 1874)

Vehilius inca (Scudder, 1872)

Vehilius stictomenes stictomenes (Butler, 1877)

Remella remus (Fabricius, 1798)

Mnasitheus sp.

Papias subcostulata (Herrich-Schäffer, 1870)

Cobalopsis cocalus (Evans, 1955)

Cobalopsis miaba (Schaus, 1902)

Cobalopsis nero (Herrich-Schäffer, 1869)

Lerema lineosa (Herrich-Schäffer, 1865)

Lerema veadeira Mielke, 1968

Morys geisa geisa (Möschler, 1878)

Morys subgrisea subgrisea (Mabille, 1898)

Cumbre belli eberti Evans, 1955

Vettius artona (Hewitson, 1868)

Vettius lafrenaye lafrenaye (Herrich-Schäffer, 1869)

Vettius lucretius (Latreille, [1824])

Vettius richardi (Weeks, 1906)

Justinia justinianus justinianus (Latreille, [1824])

Synale hylaspes (Stoll, 1781)

Synale metella (Plötz, 1882)

Argon lota (Hewitson, 1877)

Carystoides basoches (Latreille, [1824])

Perichares philetes adela (Hewitson, 1867)

Perichares seneca seneca (Latreille, [1824])

http://www.biotaneotropica.org.br 
PAPILIONIDAE Papilioninae/Graphiini

Papilioninae/Troidini

Papilioninae/Papilionini

PIERIDAE Dismorphiinae

Coliadinae
Orses cynisca (Swaison, 1821)

Quinta locutia (Hewitson, 1876)

Cynea diluta (Herrich-Schäffer, 1869)

Conga chydaea (Butler, 1877)

Copaeodes castanea Mielke, 1969

Polites vibex catilina (Plötz, 1886)

Wallengrenia otho sapuca Evans, 1955

Wallengrenia premnas (Wallengren, 1860)

Pompeius amblyspila (Mabille, 1898)

Pompeius pompeius (Latreille, [1824])

Pompeius postpuncta (Draudt, 1923)

Chalcone briquenydan briquenydan (Weeks, 1901)

Lerodea erythrostictus (Prittwitz, 1868)

Lerodea eufala eufala (Edwards, 1869)

Calpodes ethlius (Stoll, 1782)

Panoquina bola Bell, 1942

Panoquina chapada Evans, 1955

Panoquina hecebolus (Scudder, 1872)

Panoquina lucas lucas

Nyctelius nyctelius nyctelius (Latreille, [1824])

Oxynthes corusca (Herrich-Schäffer, 1869)

Xeniades chalestra chalestra (Hewitson, 1866)

Xeniades orchamus orchamus (Cramer, 1777)

Saliana longirostris (Sepp, [1840])

Pseudosarbia flavofasciata Skinner, 1921

Protesilaus protesilaus (Linnaeus, 1758)

Battus crassus crassus (Cramer, 1777)

Battus polydamas polydamas (Linnaeus, 1758)

Parides anchises foetterlei (Rothschild \& Jordan, 1906)

Heraclides anchisiades capys (Hübner, [1809])

Heraclides astyalus astyalus (Godart, 1819)

Heraclides thoas brasiliensis (Rothschild \& Jordan, 1906)

Pterourus scamander grayi (Boisduval, 1836)

Dismorphia amphione astynome (Dalman, 1823)

Dismorphia thermesia thermesia (Godart, 1819)

Enantia lina psamathe (Fabricius, 1793)

Pseudopieris nehemia nehemia (Boisduval, 1836)

Anteos clorinde (Godart, [1824])

Anteos menippe (Hübner, [1818])

Phoebis argante argante (Fabricius, 1775)

Phoebis philea philea (Linnaeus, 1763)

Phoebis sennae sennae (Linnaeus, 1758)

Rhabdodryas trite banksi (Breyer, 1939)

Aphrissa statira statira (Cramer, 1777)

Pyrisitia leuce leuce (Boisduval, 1836)

Pyrisitia nise tenella (Boisduval, 1836)

http://www.biotaneotropica.org.br 
Pierinae/Anthocharidini

Pierinae/Pierini

LYCAENIDAE Polyommatinae

Theclinae/Eumaeini
Eurema albula albula (Cramer, 1775)

Eurema agave pallida (Chavannes, 1850)

Eurema arbela arbela Geyer, 1832

Eurema deva doris (Röber, 1909)

Eurema elathea elathea (Cramer, 1777)

Eurema phiale paula (Röber, 1909)

Hesperocharis anguitia anguitia (Godart, 1819)

Cunizza hirlanda planasia Fruhstorfer, 1910

Archonias brassolis tereas (Godart, 1819)

Melete lycimnia paulista Fruhstorfer, 1908

Appias drusilla drusilla (Cramer, 1777)

Ascia monuste orseis (Godart, 1819)

Leptotes cassius cassius (Cramer, 1775)

Hemiargus hanno (Stoll, 1790)

Zizula cyna (Edwards, 1881)

Elkalyce cogina (Schaus, 1902)

Paiwarria aphaca (Hewitson, 1867)

Evenus satyroides (Hewitson, 1865)

Pseudolycaena marsyas (Linnaeus, 1758)

Theritas triquetra (Hewitson, 1865)

Theritas hemon (Cramer, 1775)

Thereus praxis (Godman \& Salvin, 1887)

Rekoa meton (Cramer, 1779)

Rekoa palegon (Cramer, 1780)

Rekoa marius (Lucas, 1857)

Arawacus ellida (Hewitson, 1867)

Arawacus aetolus (Sulzer, 1776)

Arawacus tarania (Hewitson, 1868)

Contrafacia imma (Prittwitz, 1865)

Contrafacia muattina (Schaus, 1902)

Ocaria ocrisia (Hewitson, 1868)

Chlorostrymon telea Hewitson, 1868

Magnastigma julia Nicolay, 1977

Magnastigma elsa (Hewitson, 1877)

Cyanophrys amyntor (Cramer, 1775)

Cyanophrys herodotus (Fabricius, 1793)

Bistonina mantica (Druce, 1907)

Allosmaitia strophius (Godart, [1824])

Lamprospilus badaca (Hewitson, 1868)

Arumecla galliena (Druce, 1912)

Ziegleria syllis (Godman \& Salvin, 1877)

Ziegleria perisus (Druce, 1907)

Electrostrymon endymion Fabricius, 1775

Calycopis anthora (Hewitson, 1877)

Calycopis caulonia (Hewitson, 1869)

Calycopis calor (Druce, 1907)

Calycopis demonassa (Hewitson, 1868)

http://www.biotaneotropica.org.br 
Calycopis cissusa (Hewitson, 1877) Strymon tegaea (Hewitson, 1868) Strymon mulucha (Hewitson, 1867) Strymon crambusa (Hewitson, 1874) Strymon bazochii (Godart, 1824) Strymon bubastus (Stoll, 1780) Strymon eurytulus (Hübner, [1819]) Strymon astiocha (Prittwitz, 1865) Strymon cestri (Reakirt, [1867]) Strymon ziba (Hewitson, 1868) Tmolus echion (Linnaeus, 1767) Tmolus venustus (Druce, 1907) Tmolus cydrara (Hewitson, 1868) Nicolaea cauter (Druce, 1907) Nicolaea besidia (Hewitson, 1868) Nicolaea socia (Hewitson, 1868) Nicolaea bagrada (Hewitson, 1868) Nicolaea ophia (Hewitson, 1868) Ministrymon megacles (Stoll, 1780) Ministrymon phrutus (Geyer, 1832) Ministrymon azia (Hewitson, 1873) Ministrymon cleon (Fabricius, 1775) Ministrymon una (Hewitson, 1873) Ministrymon sp.

Gargina emessa (Hewitson, 1867)

Theclopsis lydus (Hübner, [1819])

Theclopsis gargara (Hewitson, 1868)

Ostrinotes sophocles (Fabricius, 1793)

Strephonota sphinx (Fabricius, 1775)

Panthiades hebraeus (Hewitson, 1867)

Panthiades phaleros (Linnaeus, 1767)

Thepytus thyrea (Hewitson, 1867)

Oenomaus cortica (D'Abrera, 1995)

Parrhasius polibetes (Stoll, 1781)

Parrhasius orgia (Hewitson, 1867)

Michaelus thordesa (Hewitson, 1867)

Ignata mulsus (Druce, 1907)

Olynthus essus (Herrich-Schäffer, [1853])

Celmia celmus (Cramer, 1775)

Chalybs janias Hübner, 1819

Symbiopsis lenitas (Druce, 1907)

RIODINIDAE Euselasiinae/Euselasiini

Riodininae/Mesosemiini
Euselasia pellonia azurea Callaghan, 1999 Euselasia hygenius occulta Stichel, 1919 Euselasia mys cytis Stichel, 1919 Euselasia thucydides truncata Callaghan, 2001

Mesosemia melpia vaporosa Stichel, 1910

Mesosemia pardalis Callaghan, 2001

Leucochimona icare mathata (Hewitson, 1873)

Napaea eucharila parvipuncta Lathy, 1932

http://www.biotaneotropica.org.br 
Riodininae/Eurybiini

Riodininae/Riodinini

Riodininae/Symmachiini

Riodininae/Helicopini

Riodininae/Tribo Incertae Sedis

Riodininae/Nymphidiini
Eurybia halimede passercula Stichel, 1915 Alesa prema (Godart, 1824)

Lyropteryx apollonia sparsa Stichel, 1924

Lyropteryx terpsichore terpsichore Westwood, 1851 Ancyluris meliboeus meliboeus (Fabricius, 1776)

Ancyluris colubra (Saunders, 1859)

Rhetus periander arthuriana (Sharpe, 1890)

Chorinea licursis (Fabricius, 1775)

Chorinea octauius orchestris (Stichel, 1910)

Chalodeta theodora (C. Felder \& R. Felder, 1862)

Chalodeta chaonites (Hewitson, 1866)

Dachetola azora (Godart, [1824])

Pheles atricolor atricolor (Butler, 1871)

Syrmatia nyx (Hübner, [1817])

Detritivora brasilia (Harvey \& Hall, 2002)

Detritivora zama (Bates, 1868)

Detritivora gynaea (Godart, [1824])

Calephelis braziliensis McAlpine, 1971

Parcella amarynthina (C. Felder \& R. Felder, 1865)

Baeotis johannae johannae Sharpe, 1890

Lasaia agesilas agesilas (Latreille, 1809)

Amarynthis meneria (Cramer, 1776)

Riodina lycisca lycisca (Hewitson, 1853)

Melanis smithiae smithiae (Westwood, 1851)

Melanis aegates lilybaeus (Stichel, 1926)

Melanis marathon stenotaenia (Röber, 1904)

Symmachia accusatrix Westwood, 1851

Symmachia hippodice Godman, 1903

Pirascca sagaris satnius (Dalman, 1823)

Phaenochitonia fuliginea (Bates, 1868)

Stichelia bocchoris bocchoris (Hewitson, 1876)

Sarota gyas (Cramer, 1775)

Anteros formosus formosus (Cramer, 1777)

Anteros lectabilis Stichel, 1909

Emesis mandana mandana (Cramer, 1780)

Emesis diogenia Prittwitz, 1865

Emesis temesa peruviana (Lathy, 1904)

Apodemia paucipuncta Spitz, 1930

Aricoris constantius (Fabricius, 1793)

Aricoris hubrichi (Stichel, 1926)

Aricoris middletoni (Sharpe, 1890)

Aricoris caracensis Callaghan, 2001

Ariconias glaphyra (Westwood, 1851)

Lemonias theodora (Godman, 1903) 
Riodininae/Stalachtini

NYMPHALIDAE Libytheinae

Danainae/Danaini

Ithomiinae/Tithoreini

Ithomiinae/Mechanitini

Ithominae/Napeogenini

Ithomiinae/Ithomiini

Ithomiinae/Oleriinii

Ithomiinae/Dircennini

Ithomiinae/Godyridini
Thisbe hyalina (Butler, 1867)

Juditha molpe (Hübner, [1808])

Synargis calyce (C. Felder \& R. Felder, 1862)

Synargis agle (Hewitson, [1853])

Synargis chaonia (Hewitson, [1853])

Synargis ethelinda (Hewitson, 1870)

Synargis paulistina (Stichel, 1910)

Synargis galena (Bates, 1868)

Synargis axenus axenus (Hewitson, 1876)

Adelotypa zerna (Hewitson, 1872)

Nymphidium leucosia leucosia (Hübner, [1806])

Nymphidium lisimon (Stoll, 1790)

Theope eudocia Westwood, 1851

Theope thootes (Hewitson, 1860)

Theope thestias thestias (Hewitson, 1860)

Theope foliorum Bates, 1868

Stalachtis phlegia phlegetontia (Perty, 1833)

Stalachtis susanna (Fabricius, 1787)

Libytheana carinenta (Cramer, 1779)

Danaus gilippus gilippus (Cramer, 1775)

Danaus eresimus plexaure (Godart, 1819)

Danaus erippus (Cramer, 1775)

Lycorea halia discreta Haensch, 1909

Tithorea harmonia pseudethra Butler, 1873

Aeria olena Weymer, 1875

Aeria elara elarina (Oberthür, 1879)

Methona themisto (Hübner, 1818)

Thyridia psidii hippodamia (Fabricius, 1775)

Mechanitis lysimnia lysimnia (Fabricius, 1793)

Mechanitis polymnia casabranca Haensch, 1905

Hypothyris euclea laphria (Doubleday, 1847)

Hypothyris ninonia daeta (Boisduval, 1836)

Ithomia agnosia agnosia (Hewitson, [1855])

Placidina euryanassa (C. Felder \& R. Felder, 1860)

Oleria aquata (Weymer, 1875)

Dircenna dero dero (Hübner, 1823)

Episcada hymenaea centralis (Brown \& Mielke 1970)

Hypoleria lavinia consimilis Talbot, 1928

Hypoleria sarepta goiana d'Almeida, 1951

Brevioleria aelia plisthenes (d'Almeida, 1958)

Brevioleria seba emyra (Haensch, 1905)

http://www.biotaneotropica.org.br 
Morphinae/Morphini

Morphinae/Brassolini

Satyrinae/Satyrini
Mcclungia cymo salonina (Hewitson, 1855)

Heterosais edessa (Hewitson, [1855])

Pseudoscada erruca (Hewitson, 1855)

Pseudoscada acilla quadrifasciata Talbot, 1928

Morpho menelaus coeruleus (Perry, 1810)

Morpho helenor achillides (C. Felder \& R. Felder, 1867)

Narope cyllabarus Westwood, 1851

Narope cyllarus Westwood, 1851

Brassolis sophorae laurentii Stichel, 1925

Brassolis sophorae sophorae (Linnaeus, 1758)

Dynastor darius darius (Fabricius, 1775)

Blepolenis batea ssp. $\mathrm{n}$.

Opsiphanes cassiae crameri C. Felder \& R. Felder, 1862

Opsiphanes invirae remoliatus Fruhstorfer, 1907

Catoblepia berecynthia (Cramer, 1777)

Eryphanis automedon automedon (Cramer, 1775)

Eryphanis reevesii reevesii (Doubleday, [1849])

Caligo illioneus illioneus (Cramer, 1775)

Pampasatyrus ocelloides (Schaus, 1902)

Cercyeuptychia luederwaldti (Spitz, 1931)

Cissia terrestris (Butler, 1866)

Erichthodes narapa (Schaus, 1902)

Euptychia westwoodi Butler, 1867

Godartiana muscosa (Butler, 1870)

Hermeuptychia hermes (Fabricius, 1775)

Hermeuptychia fallax (C. Felder \& R. Felder, 1862)

Magneuptychia ocnus (Butler, 1867)

Pareuptychia ocirrhoe ocirrhoe (Fabricius, 1777)

Pareuptychia summandosa (Gosse, 1880)

Paryphthimoides numeria (C. Felder \& R. Felder, 1867)

Paryphthimoides undulata (Butler, 1867)

Paryphthimoides phronius (Godart, [1824])

Paryphthmoides poltys (Prittwitz, 1865)

Pharneuptychia innocentia (C. Felder \& R. Felder, 1867)

Pharneuptychia phares (Godart, [1824])

Praefaunula armilla (Butler, 1867)

Taygetis chiquitana Forster, 1964

Taygetis echo (Cramer, 1775)

Taygetis kerea Butler, 1869

Taygetis laches (Fabricius, 1793)

Taygetis mermeria mermeria (Cramer, 1776)

Taygetis virgilia (Cramer, 1776)

Taygetomorpha celia (Cramer, 1779)

Yphthimoides argyrospila (Butler, 1867)

Yphthimoides ochracea (Butler, 1867)

Yphthimoides pacta (Weymer, 1911)

Yphthimoides straminea (Butler, 1867)

Yphthimoides yphthima (C. Felder \& R. Felder, 1867) 
Yphthimoides celmis (Godart, [1824])

Yphthimoides maepius maepius (Godart, [1824])

Yphthimoides renata (Stoll, 1780)

Satyrinae/Tribo Incertae sedis

Charaxinae/Anaeini

Charaxinae/Preponini

Biblidinae/Cyrestini

Biblidinae/Biblidini
Amphidecta reynoldsi reynoldsi Sharpe, 1890

Siderone galanthis (Cramer, 1775)

Zaretis isidora (Cramer, 1779)

Fountainea glycerium cratias (Hewitson, 1874)

Fountainea ryphea phidile (Geyer, 1837)

Memphis acidalia victoria (Druce, 1877)

Memphis moruus stheno (Prittwitz, 1865)

Archaeoprepona amphimachus amphimachus (Fabricius, 1775) Archaeoprepona demophon thalpius (Hübner, [1814]) Archaeoprepona demophoon antimache (Hübner, [1819]) Prepona dexamenus dexamenus Hopffer, 1874 Prepona laertes demodice (Godart, [1824])

Prepona pheridamas (Cramer, 1777)

Agrias claudina godmani Fruhstorfer, 1895

Marpesia chiron (Fabricius, 1775)

Marpesia petreus petreus (Cramer, 1776)

Biblis hyperia nectanabis (Fruhstorfer, 1909) Mestra dorcas apicalis (Staudinger, 1886)

Catonephele acontius caeruleus (Jenkins, 1985)

Catonephele numilia penthia (Hewitson, 1852)

Eunica bechina (Hewitson, 1852)

Eunica eburnea Fruhstorfer, 1907

Eunica cuvierii (Godart, 1819)

Eunica tatila bellaria Fruhstorfer, 1908

Eunica volumna volumna (Godart, 1824])

Hamadryas laodamia laodamia (Cramer, 1777)

Hamadryas amphinome amphinome (Linnaeus, 1767)

Hamadryas februa (Hübner, [1823])

Hamadryas feronia feronia (Linnaeus, 1758)

Hamadryas iphthime iphthime (Bates, 1864)

Hamadryas chloe rhea (Fruhstorfer, 1907)

Epiphile orea (Hübner, [1823])

Nica flavilla flavilla (Godart, [1824])

Temenis laothoe ssp.

Temenis huebneri korallion Fruhstorfer, 1912

Dynamine postverta postverta (Cramer, 1779)

Dynamine tithia tithia (Hübner, [1823])

Dynamine agacles agacles (Dalman, 1823)

Callicore astarte selima (Guenée,1872)

Callicore sorana sorana (Godart, [1824])

Diaethria clymena janeira (C. Felder, 1862)

Diaethria eluina eluina Hewitson, [1855]) 
Apaturinae

Nymphalinae/Coeini

Nymphalinae/Nymphalini

Nymphalinae/Kallimini

Nymphalinae/Melitaeini

Limenitidinae/Limenitidini

Heliconiinae/Argynnini

Heliconiinae/Acraeini

Heliconiinae/Heliconiini
Doxocopa laurentia (Godart, [1824])

Colobura dirce dirce (Linnaeus, 1758)

Historis acheronta acheronta (Fabricius, 1775)

Historis odius dious Lamas, 1995

Smyrna blomfildia blomfildia (Fabricius, 1781)

Tigridia acesta latifascia (Butler, 1873)

Hypanartia lethe (Fabricius, 1793)

Vanessa braziliensis (Moore, 1883)

Vanessa myrinna (Doubleday, 1849)

Junonia evarete evarete (Cramer, 1779)

Anartia amathea roeselia (Eschscholtz, 1821)

Anartia jatrophae jatrophae (Linnaeus, 1763)

Siproeta stelenes stelenes (Linnaeus, 1758)

Siproeta epaphus trayja Hübner, [1823]

Chlosyne lacinia saundersi (Doubleday, [1847])

Anthanassa frisia hermas (Hewitson, 1864)

Eresia eunice esora Hewitson, 1857

Eresia lansdorfi (Godart, 1819)

Ortilia dicoma (Hewitson, 1864)

Ortilia ithra (Kirby, 1900)

Ortilia sejona (Schaus, 1902)

Tegosa claudina (Eschscholtz, 1821)

Adelpha iphiclus ephesa (Ménétriés, 1857)

Adelpha malea aethalia (C. Felder \& R. Felder, 1867)

Adelpha plesaure phliassa (Godart [1824])

Adelpha cytherea aea (C. Felder \& R. Felder, 1867)

Adelpha abia (Hewitson, 1850)

Adelpha paraena paraena (Bates, 1865)

Adelpha thoasa gerona (Hewitson, 1867)

Euptoieta hegesia meridiania Stichel, 1938

Actinote carycina Jordan, 1913

Actinote discrepans d'Almeida 1958

Actinote parapheles Jordan, 1913

Actinote pellenea pellenea Hübner, [1821]

Actinote rhodope d'Almeida, 1923

Actinote surima surima (Schaus, 1902)

Agraulis vanillae maculosa (Stichel, [1908])

Dione juno suffumata (Brown \& Mielke, 1972)

Dryadula phaetusa (Linnaeus, 1758)

Dryas iulia alcionea (Cramer, 1779)

Eueides isabella dianasa (Hübner, [1806])

Eueides aliphera aliphera (Godart, 1819)

Heliconius ethilla narcea Godart, 1819

http://www.biotaneotropica.org.br 
Heliconius besckei Ménétriés, 1857

Heliconius erato phyllis (Fabricius, 1775)

Heliconius melpomene burchelli Poulton, 1910

Heliconius sara thamar (Hübner, [1806]) 